\title{
Robust Distributed Model Predictive Control of Linear Systems
}

\author{
Christian Conte ${ }^{1}$, Melanie N. Zeilinger ${ }^{2}$, Manfred Morari ${ }^{1}$ and Colin N. Jones ${ }^{2}$
}

\begin{abstract}
In this work, synthesis and closed-loop operation of robust distributed model predictive control (MPC) for linear systems using distributed optimization is discussed. Previous work has shown that a nominal MPC controller for this setup can be synthesized and operated in a purely distributed manner. This paper extends this concept to linear systems subject to additive bounded disturbance. It is shown how well-established robust MPC approaches can be applied to distributed systems. The main focus of the paper is on a thorough discussion of computational issues arising from distributed synthesis and closed-loop operation of existing robust MPC controllers. In particular, techniques for distributed synthesis of structured robust positive invariant sets and distributed constraint tightening are proposed. The paper is concluded by a numerical example which illustrates the functionality and performance of the proposed techniques.
\end{abstract}

\section{INTRODUCTION}

The main challenge in the formulation of control strategies for distributed dynamic systems is the fact that communication among subsystems is limited. This property applies to systems from numerous fields, such as power systems [1] or irrigation canals [2]. Due to the presence of state and input constraints in such systems, distributed MPC is an attractive control scheme.

Distributed MPC has been an area of active research in the last decade. The approaches proposed in the literature can be categorized into two classes: iterative and non-iterative. In iterative distributed MPC, the subsystems do multiple communication rounds per time step, while in non-iterative distributed MPC they communicate only once. Most iterative approaches rely on distributed optimization, e.g. [3], [4]. Thus, all subsystems collaboratively follow a global performance objective. Contrary, most non-iterative approaches rely on locally constrained controllers which are made robust against dynamic coupling effects [5], [6]. As a consequence, in the presence of strong coupling, the iterative approach is advantageous since the synthesis of non-iterative controllers might yield conservative control laws or even be infeasible. While nominal iterative distributed MPC was a field of active recent research [7], [8], [9], the robust case has received little attention and is therefore discussed in this paper.

In particular, this paper extends the work on nominal distributed MPC presented in [9] to linear systems subject to

The research leading to these results has received funding from the European Union Seventh Framework Programme FP7/2007-2013 under grant agreement number FP7-ICT-2009-4 248940.

${ }^{1}$ Automatic Control Laboratory, Department of Information Technology and Electrical Engineering, ETH Zurich, 8092 Zurich, Switzerland cconte |morariccontrol. ee. ethz.ch

${ }^{2}$ Automatic Control Laboratory, École Polytechnique Fédérale de Lausanne (EPFL), 1015 Lausanne, Switzerland melanie.zeilinger|colin. jonesdepfl.ch bounded additive disturbance and shows how existing robust MPC formulations for centralized MPC can be applied in a distributed way. Existing formulations are usually either of min-max type [10], or based on tubes [11]. Due to the high computational complexity inherent to min-max approaches, we focus on tube MPC is considered in this paper. Distributed tube MPC has been considered in [6] for a non-iterative parallel, and in [12] for a non-iterative sequential setup.

In contrast, this paper considers tube techniques tailored for iterative distributed MPC based on distributed optimization. The paper highlights computational issues, which arise if existing tube MPC controllers are synthesized and computed on a distributed system. As a first contribution of the paper, it is shown how structured versions of these controllers can be synthesized in a distributed manner. The main synthesis steps include constraint tightening and distributed computation of robust positive invariant sets. As a second contribution, it is to shown that the resulting structured controllers can be operated using distributed optimization in closed-loop.

The paper is structured as follows: In Section II all necessary preliminaries on distributed systems and optimization are given. In Section III, the two particular robust MPC approaches used are outlined. Section IV discusses distributed synthesis of these approaches, while Section V discusses distributed closed-loop operation. All approaches are compared in a numerical example in Section VI.

\section{Preliminaries}

\section{A. Notation}

A block-diagonal matrix with blocks $S_{i}, i \in\{1, \ldots, M\}$, is denoted by $\operatorname{diag}_{i \in\{1, \ldots, M\}}\left(S_{i}\right)$, a vector consisting of stacked sub vectors $s_{i}, i \in\{1, \ldots, M\}$, is denoted by $\operatorname{col}_{i \in\{1, \ldots, M\}}\left(s_{i}\right)$. Furthermore, for a set $\mathcal{S} \subseteq \mathbb{R}^{n}$ and a matrix $T \subseteq \mathbb{R}^{l \times n}$, the set $T \mathcal{S}$ is defined as $\left\{x \in \mathbb{R}^{l} \mid \exists s \in\right.$ $\mathcal{S}: x=T s\}$. For two sets $\mathcal{S}, \mathcal{T} \subseteq \mathbb{R}^{n}, \mathcal{S} \oplus \mathcal{T}=\{x \in$ $\left.\mathbb{R}^{n} \mid \exists s \in \mathcal{S}, t \in \mathcal{T}: x=s+t\right\}$ denotes the Minkowski sum and $\mathcal{S} \ominus \mathcal{T}=\left\{x \in \mathbb{R}^{n} \mid x+t \in \mathcal{S}, \forall t \in \mathcal{T}\right\}$ denotes the Pontryagin difference.

\section{B. Distributed Linear Systems}

We consider a network of $M$ interconnected linear subsystems of the form

$$
x_{i}^{+}=\left(\sum_{j=1}^{M} A_{i j} x_{j}\right)+B_{i} u_{i}+w_{i},
$$

where state and input of each subsystem $i$ are denoted by $x_{i} \in \mathbb{R}^{m_{i}}$ and $u_{i} \in \mathbb{R}^{p_{i}}$ respectively. Moreover, $w_{i} \in \mathbb{R}^{m_{i}}$ is 
a bounded disturbance entering the dynamics of subsystem $i$. Communication in the network is assumed to be limited to neighboring subsystems.

Definition II.1 (Neighboring Subsystems) Subsystem $j$ is a neighbor of subsystem $i$ if $A_{i j} \neq 0 . B y \mathcal{N}_{i}$ we denote the set of subsystem $i$ and its neighbors. Moreover, by $x_{\mathcal{N}_{i}}=\operatorname{col}_{j \in \mathcal{N}_{i}}\left(x_{j}\right) \in \mathbb{R}^{m_{\mathcal{N}_{i}}}$, we denote the vector of all states belonging to subsystems in $\mathcal{N}_{i}$.

The dynamics of subsystem $i$ can thus be alternatively described as

$$
x_{i}^{+}=A_{\mathcal{N}_{i}} x_{\mathcal{N}_{i}}+B_{i} u_{i},
$$

where $A_{\mathcal{N}_{i}} \in \mathbb{R}^{m_{i} \times m_{\mathcal{N}_{i}}}$.

Assumption II.2 (Communication) Two subsystems $i$ and $j$ can communicate in a bidirectional way if $i \in \mathcal{N}_{j} \vee j \in \mathcal{N}_{i}$.

The dynamics of the global network of subsystems are described by the linear system

$$
x^{+}=A x+B u+w,
$$

where $A$ is block-sparse with non-zero blocks $A_{i j}$ and $B=\operatorname{diag}\left(B_{1}, \ldots, B_{M}\right)$ is block-diagonal. Furthermore, $x=\operatorname{col}_{i \in\{1, \ldots, M\}}\left(x_{i}\right) \in \mathbb{R}^{m}$ is the global state, $u=$ $\operatorname{col}_{i \in\{1, \ldots, M\}}\left(u_{i}\right) \in \mathbb{R}^{p}$ the global input and $w=$ $\operatorname{col}_{i \in\{1, \ldots, M\}}\left(w_{i}\right) \in \mathbb{R}^{m}$ the global disturbance. State and input are constrained to lie in convex compact sets

$$
\begin{aligned}
& \mathcal{X}=\mathcal{X}_{1} \times \ldots \times \mathcal{X}_{M} \\
& \mathcal{U}=\mathcal{U}_{1} \times \ldots \times \mathcal{U}_{M}
\end{aligned}
$$

where for each subsystem $i, \mathcal{X}_{i} \subseteq \mathbb{R}^{m_{i}}$ and $\mathcal{U}_{i} \subseteq \mathbb{R}^{p_{i}}$ contain the origin in their interior. Moreover, the global disturbance $w$ is assumed to lie in a convex compact set

$$
\mathcal{W}=\mathcal{W}_{1} \times \ldots \times \mathcal{W}_{M}
$$

where for each subsystem $i, \mathcal{W}_{i} \subseteq R^{m_{i}}$ contains the origin. For system (3), existence of a stabilizing structured control law is assumed.

Assumption II.3 (Structured Control Law) There exists a stabilizing linear state feedback control law with distributed structure for system (3), i.e. $u=K_{t} x=$ $\operatorname{col}_{i \in\{1, \ldots, M\}}\left(K_{t, i} x_{\mathcal{N}_{i}}\right)$, where $K_{t, i} \in \mathbb{R}^{p_{i} \times m_{\mathcal{N}_{i}}}$. Distributed techniques to find such structured control laws are described for instance in [9].

Two classes of invariant sets are defined in the following:

Definition II.4 (Positively invariant (PI) set) A set $\mathcal{S} \in$ $\mathbb{R}^{n}$ is a positively invariant (PI) set for system $x^{+}=$ $A x+B \kappa(x)$, if $x^{+} \in \mathcal{S}$ for all $x \in \mathcal{S}$.

Definition II.5 (Robust positively invariant (RPI) set) $A$ set $\mathcal{S}$ is a robust positively invariant (RPI) set for system $x^{+}=A x+B \kappa(x)+w$, if $x^{+} \in \mathcal{S}$ for all $x \in \mathcal{S}, w \in \mathcal{W}$. $\mathcal{S}_{\text {min }}$ is the minimum RPI set if for any RPI set $\mathcal{S}$ it holds that $\mathcal{S}_{\text {min }} \subseteq \mathcal{S}$.

\section{Distributed Optimization}

Distributed optimization is a key component for both synthesis and closed-loop operation of iterative distributed MPC as presented in this work. In particular, it allows the application of well-established MPC theory (see e.g. [13]) to distributed systems. The main requirements for distributed optimization to be of use for MPC is for the global MPC problem to be structured and for Assumption II.2 to hold. A detailed discussion of distributed optimization concepts and methods is beyond the scope of this paper, the reader is referred to the standard reference [14] instead.

\section{RoBUST MPC}

In this paper, we focus on the two robust MPC approaches presented in [11] and [15] respectively. In the approach proposed in [11], the nominal constraints are tightened by an RPI set, whereas in the approach proposed in [15], they are tightened over the MPC prediction horizon. Furthermore, in [15], pre-stabilized dynamics are used. In the next two sections, we briefly outline the two approaches in order to then propose methods for distributed synthesis and closedloop operation in Section IV and Section V.

\section{A. Robust MPC according to [11]}

As a first approach, robust MPC according to [11] is considered. In this approach, it is ensured that the perturbed system state remains in a tube around a nominally stabilizing trajectory, and the constraints on this nominal trajectory are tightened by the cross section of the tube. This cross section is specifically chosen to be an RPI set $\mathcal{Z}$ for the system $x^{+}=\left(A+B K_{t}\right) x+w$. The nominally stabilizing trajectory is obtained by solution of the MPC problem

$$
\begin{aligned}
& V_{r 1}(x)=\min _{x(0), \mathbf{u}} \sum_{k=0}^{N-1} l(x(k), u(k))+V_{f}(x(N)) \\
& \text { s.t. } x(0) \in x \oplus \mathcal{Z}, \\
& \quad x(k+1)=A x(k)+B u(k) \forall k \in\{0, . ., N-1\}, \\
& \quad(x(k), u(k)) \in \overline{\mathcal{X}} \times \overline{\mathcal{U}} \forall k \in\{0, . ., N-1\}, \\
& \quad x(N) \in \overline{\mathcal{X}}_{f}
\end{aligned}
$$

where

$$
\overline{\mathcal{X}}=\mathcal{X} \ominus \mathcal{Z}, \overline{\mathcal{U}}=\mathcal{U} \ominus K_{t} \mathcal{Z}, \overline{\mathcal{X}}_{f} \subseteq \overline{\mathcal{X}}
$$

In (7), $\mathbf{u}=\{u(0), \ldots, u(N-1)\}$ is the sequence of control inputs which uniquely defines the sequence of states $\mathbf{x}=\{x(0), \ldots, x(N)\}$ given $x(0)$. Moreover, $\overline{\mathcal{X}}_{f}$ is a PI set and $\mathcal{X}_{N}^{r 1}$ denotes the set of states $x$, for which there exists a feasible solution to problem (7). Finally, the robust constrained control law is defined as

$$
\kappa_{r 1}(x):=u_{r 1}^{*}(0)+K_{t}\left(x-x_{r 1}^{*}(0)\right),
$$

where $x_{r 1}^{*}(0)$ and $u_{r 1}^{*}(0)$ are the first elements of the optimal state and input trajectories for problem (7). Note that due to constraint (7b), the initial state of the nominal trajectory does not generally coincide with the current system state. 
Theorem III.1 (Theorem 1 in [11]) Given the existence of a control law $\kappa_{f}(x)$, such that $\forall x \in \overline{\mathcal{X}}_{f} \subseteq \overline{\mathcal{X}}$ :

$$
\begin{aligned}
& \kappa_{f}(x) \in \overline{\mathcal{U}}, \quad A x+B \kappa_{f}(x) \in \overline{\mathcal{X}}_{f} \\
& V_{f}\left(A x+B \kappa_{f}(x)\right)-V_{f}(x) \leq-l\left(x, \kappa_{f}(x)\right),
\end{aligned}
$$

the state of system $x^{+}=A x+B \kappa_{r 1}(x)+w, w \in \mathcal{W}$, exponentially converges to $\mathcal{Z}$ with region of attraction $\mathcal{X}_{N}^{r 1}$.

\section{B. Robust MPC according to [15]}

As a second robust MPC formulation, the approach proposed in [15] is considered. This is a standard constraint tightening approach, where the dynamics are pre-stabilized as $x^{+}=\left(A+B K_{t}\right) x+w$ in order to introduce feedback on the disturbance. A nominal state trajectory is obtained by solving the MPC problem

$$
\begin{aligned}
& V_{r 2}(x)=\min _{\mathbf{v}} \sum_{k=0}^{N-1} l(x(k), v(k))+V_{f}(x(N)) \\
& \text { s.t. } x(0)=x \\
& \quad x(N) \in \overline{\mathcal{X}}_{f} \\
& \forall k \in\{0, \ldots, N-1\}: \\
& \quad x(k+1)=\left(A+B K_{t}\right) x(k)+B v(k) \\
& \quad\left(x(k), v(k)+K_{t} x(k)\right) \in \overline{\mathcal{X}}_{k} \times \overline{\mathcal{U}}_{k} .
\end{aligned}
$$

In problem (12), the constraints are tightened at each prediction time as

$$
\begin{aligned}
& \overline{\mathcal{X}}_{k}=\mathcal{X} \ominus\left(\bigoplus_{j=0}^{k-1}\left(A+B K_{t}\right)^{j} \mathcal{W}\right), \\
& \overline{\mathcal{U}}_{k}=\mathcal{U} \ominus K_{t}\left(\bigoplus_{j=0}^{k-1}\left(A+B K_{t}\right)^{j} \mathcal{W}\right),
\end{aligned}
$$

where $\overline{\mathcal{X}}_{f} \subseteq \overline{\mathcal{X}}_{N}$ and $\overline{\mathcal{X}}_{f}$ is a PI set. Furthermore, the set $\mathcal{X}_{N}^{r 2}$ denotes the set of parameters $x$, for which there exists a feasible solution to problem (12). Finally, the control law derived from problem (12) is defined as

$$
\kappa_{r 2}(x):=v_{r 2}^{*}(0)+B K_{t} x,
$$

where $v_{r 2}^{*}(0)$ is the first element of the optimal variable sequence in the sense of problem (12). Note that under the control law (15), problem (12) is recursively feasible.

Theorem III.2 (Theorem 8 in [15]) The state of system $x^{+}=A x+B \kappa_{r 2}(x)+w$ converges to the minimum $R P I$ set of system $x^{+}=\left(A+B K_{t}\right) x+w, w \in \mathcal{W}$, with region of attraction $\mathcal{X}_{N}^{r 2}$.

\section{Robust MPC: Distributed Synthesis}

The synthesis of the two robust MPC controllers introduced in Section III depends on the following operations:

1) Synthesis of a terminal cost $V_{f}(x)$ and a set $\overline{\mathcal{X}}_{f}$.

2) Synthesis of an RPI set $\mathcal{Z}$.

3) Constraint tightening, i.e. operations (8) and operations (13) and (14) respectively.
For networks of dynamic systems, which might be subject to online changes in topology, it is important to have the possibility of distributed controller synthesis. Distributed synthesis of structured terminal costs $V_{f}(x)$ and terminal sets $\mathcal{X}_{f}$ was studied in [9]. For the synthesis of $\mathcal{Z}$ and for constraint tightening, however, only centralized methods exist, e.g. [16]. This section focuses on distributed methods for the solution of those two problems. In particular, distributed computation of RPI sets is discussed in Section IV-A and distributed constraint tightening is discussed in Section IV-B. The main challenge is the fact that both operations involve the solution of infinite horizon problems, for which information of all subsystems have to be considered.

In order to simplify the discussion, we focus on polytopic state and input constraints of the form

$$
\mathcal{X}=\left\{x \in \mathbb{R}^{m} \mid G x \leq g\right\}, \mathcal{U}=\left\{u \in \mathbb{R}^{p} \mid H u \leq h\right\},
$$

where $G \in \mathbb{R}^{q \times m}$ and $H \in \mathbb{R}^{r \times p}$. Note that both $G$ and $H$ are block-diagonal due to the decomposable structure of state and input constraints assumed in (4). Furthermore, for every subsystem $i$, the local disturbance is assumed to lie in a ball

$$
\mathcal{W}_{i}=\left\{w_{i} \in \mathbb{R}^{m_{i}} \mid w_{i}^{T} w_{i} \leq v_{i}\right\} .
$$

\section{A. Distributed Synthesis of Structured RPI Sets}

For centralized linear systems, the problem of synthesizing RPI sets has been extensively studied, see e.g. [16]. It is well known, that the minimum RPI set for a given closed-loop system $x^{+}=\left(A+B K_{t}\right) x+w$ is given by

$$
\mathcal{Z}_{\text {min }}=\bigoplus_{k=0}^{\infty}\left(A+B K_{t}\right)^{k} \mathcal{W}
$$

Definition (18) illustrates a fundamental difficulty in the synthesis of RPI sets for distributed systems: Any RPI set is defined globally since the infinite horizon in (18) couples all subsystem in the network. Synthesis of a structured RPI set is therefore a global problem and in this section, a distributed way to solve it is proposed. While for linear systems, synthesis methods for both polyhedral and ellipsoidal RPI sets exist, only ellipsoidal sets are considered in this paper due to better scalability properties of the corresponding synthesis methods.

An ellipsoidal RPI set of the form $\mathcal{Z}=\left\{x \in \mathbb{R}^{m} \mid x^{T} P x \leq\right.$ 1) can be synthesized using the S-procedure (see [17] for details), which results in an LMI encoding the implication

$$
\left.\begin{array}{rl}
x^{T} P x & \leq 1 \\
w^{T} w & \leq v
\end{array}\right\} \Rightarrow x^{+, T} P x^{+} \leq 1
$$

where $x^{+}=\left(A+B K_{t}\right) x+w$. For robust distributed MPC, a structured RPI set is defined as follows.

Definition IV.1 (Structured RPI Set) An RPI set $\mathcal{Z} \subseteq \mathbb{R}^{m}$ is structured if $\mathcal{Z}=\left\{x \in \mathbb{R}^{m} \mid x^{T} P x \leq 1\right\}$ with $x^{T} \bar{P} x=$ $\sum_{i=1}^{M} x_{\mathcal{N}_{i}}^{T} P_{\mathcal{N}_{i}} x_{\mathcal{N}_{i}}$ and all $P_{\mathcal{N}_{i}}$ positive semi-definite.

Given a structured RPI set $\mathcal{Z}$, local sets of the form $\mathcal{Z}_{i}=$ $\left\{x_{\mathcal{N}_{i}} \in \mathbb{R}^{m_{\mathcal{N}_{i}}} \mid x_{\mathcal{N}_{i}}^{T} P_{\mathcal{N}_{i}} x_{\mathcal{N}_{i}} \leq \beta_{i}\right\}$ can be defined and $x \in \mathcal{Z}$ 
is equivalent to the condition

$$
\forall i \in\{1, \ldots, M\} \exists \beta_{i} \geq 0: x_{\mathcal{N}_{i}} \in \mathcal{Z}_{i}, \sum_{i=1}^{M} \beta_{i} \leq 1 .
$$

Thus, condition (20) allows for a decomposition of $x \in \mathcal{Z}$ into local constraints which are coupled by only a single global constraint. Constraints of this kind can be handled by distributed consensus optimization as pointed out in [18].

For the synthesis of structured RPI sets, the global Sprocedure encoding the implication

$$
\left.\begin{array}{rl}
x^{T} P x & \leq 1 \\
w_{i}^{T} w_{i} & \leq v_{i} \forall i \in\{1, \ldots, M\}
\end{array}\right\} \Rightarrow x^{+T} P x^{+} \leq 1
$$

is used, where $x^{+}=\left(A+B K_{t}\right) x+w$. The LMI resulting from the S-procedure (21) is stated in (24), where all elements denoted with a bar have been lifted to fit the global variable space. Although decomposition of (24) is not straightforward, a sufficient condition can be derived and is given by the set of LMIs (25), in combination with the constraint

$$
\sum_{i=1}^{M} \bar{S}_{i} \geq 0
$$

Both in (24) and in (25) it holds that $s_{i} \geq 0$ for all $i \in$ $\{1, \ldots, M\}$.

In order to obtain the desired structured RPI set, a synthesis problem of the form

$$
\begin{aligned}
& \min _{P} g_{P}(P) \\
& \text { s.t. } \\
& \quad P_{\mathcal{N}_{i}} \geq 0 \forall i \in\{1, \ldots, M\}, \\
& \quad P=\sum_{i=1}^{M} \bar{P}_{\mathcal{N}_{i}}
\end{aligned}
$$

is solved. In (23), constraint (23b) is a sufficient condition for implication (21) to hold, constraint (23c) ensures that the submatrices are positive semi-definite and (23d) ensures the structure of the resulting global matrix $P$. The objective function $g_{P}(P)$ is a design parameter and should be chosen such that it is decomposable and enforces the intended shape and size of the resulting RPI set (e.g. $g_{P}(P)=\sum_{i=1}^{M} g_{i}\left(P_{\mathcal{N}_{i}}\right)$, where $g_{i}\left(P_{\mathcal{N}_{i}}\right)$ penalizes the norm difference of $P_{\mathcal{N}_{i}}$ to a reference matrix).

Remark IV.2 A structured RPI set $\mathcal{Z}$ can also be synthesized to be completely decentralized. Specifically, consider local sets $\mathcal{Z}_{i}=\left\{x_{i} \in \mathbb{R}^{m_{i}} \mid x_{i}^{T} P_{i} x_{i} \leq 1\right\}$, which fulfill the conditions

$$
\begin{aligned}
& \forall i \in\{1, \ldots, M\} \quad: \\
& \left.\begin{array}{rl}
x_{j}^{T} P_{j} x_{j} & \leq 1 \forall j \in \mathcal{N}_{i} \\
w_{i}^{T} w_{i} & \leq v_{i}
\end{array}\right\} \Rightarrow x_{i}^{+T} P_{i} x_{i}^{+} \leq 1,
\end{aligned}
$$

where $x_{i}^{+}=\left(A_{\mathcal{N}_{i}}+B_{i} K_{t, i}\right) x_{\mathcal{N}_{i}}+w_{i}$. Conditions (26) can conveniently be modelled by $S$-procedures, which results in a system of $M$ coupled LMIs. These LMIs can then be solved by distributed optimization. This approach is expected to be more conservative than the one based on structured RPI sets since the local sets $\mathcal{Z}_{i}$ are not only robust against local disturbance but also against dynamic coupling.

\section{B. Distributed Constraint Tightening}

In the following, a distributed method for tightening of local polyhedral constraints by a structured ellipsoidal RPI set is proposed in Section IV-B.1. Furthermore, a distributed method for tightening of local polyhedral constraints over a finite horizon is proposed in Section IV-B.2.

1) Distributed Tightening of Polytopic Constraints by an Ellipsoidal RPI Set: Tightening of local polytopic state and input constraints by a structured ellipsoidal set is required for robust distributed MPC based on [11], as stated in (8). A tightening of this kind can be achieved by the support function.

Definition IV.3 (Support function) The support function $\sigma_{\mathcal{Z}}(\cdot): \mathbb{R}^{m} \rightarrow \mathbb{R}_{+}$is defined as

$$
\sigma_{\mathcal{Z}}(a)=\sup _{x \in \mathcal{Z}} a^{T} x
$$

A tightened state constraint set $\overline{\mathcal{X}}=\left\{x \in \mathbb{R}^{m} \mid G x \leq \bar{g}\right\}$, as well as a tightened input constraint set, i.e. $\overline{\mathcal{U}}=\{u \in$ $\left.\mathbb{R}^{p} \mid H u \leq \bar{h}\right\}$, can thus be obtained by modifying every single half space constraint as

$$
\begin{aligned}
& \bar{g}_{j}=g_{j}-\sigma_{\mathcal{Z}}\left(G_{j}^{T}\right) \forall j \in\{1, \ldots, q\}, \\
& \bar{h}_{l}=h_{l}-\sigma_{\mathcal{Z}}\left(K_{t}^{T} H_{l}^{T}\right) \forall l \in\{1, \ldots, r\},
\end{aligned}
$$

where $G_{j}$ and $H_{l}$ are the $j$ 'th and $l$ 'th row of the matrices $G$ and $H$ as defined in (16). As pointed out in Section IV-A, the global constraint $x \in \mathcal{Z}$ can be handled by distributed consensus optimization if $\mathcal{Z}$ is structured. Thus, the evaluation of the support function in (28) and (29) can be performed in a distributed way for every local polyhedral constraint.

2) Distributed Tightening of Polytopic Constraints over a k-step Horizon: Tightening of local polytopic state and input constraints over a $k$-step horizon is required for robust distributed MPC based on [15]. The tightening operations are defined in (13) and (14) and can be executed by use of the $\mathrm{k}$-step support function, which is defined in the following:

Definition IV.4 (k-step support function) The $k$-step support function is defined as

$$
\begin{aligned}
\sigma_{\mathcal{W}}(a, k)=\sup _{\boldsymbol{w} \in \mathcal{W}^{k}} a^{T} y(k) & \\
\text { s.t. } \quad & y(0)=0 \\
& y(l+1)=\left(A+B K_{t}\right) y(l)+w(l) \\
& \forall l \in\{0, \ldots, k-1\},
\end{aligned}
$$

where $\mathcal{W}^{k}$ denotes the Cartesian product $\prod_{i=0}^{k-1} \mathcal{W}$ and where $\boldsymbol{w}$ is a sequence of $k$ disturbance realizations.

A $k$-step tightened state constraint set, i.e. $\overline{\mathcal{X}}_{k}=\{x \in$ $\left.\mathbb{R}^{m} \mid G x \leq \bar{g}_{k}\right\}$, as well as a $k$-step tightened input constraint 


$$
\begin{aligned}
& {\left[\begin{array}{ccc}
-\left(A+B K_{t}\right)^{T} \sum_{i=1}^{M} \bar{P}_{\mathcal{N}_{i}}\left(A+B K_{t}\right) & -\left(A+B K_{t}\right)^{T} \sum_{i=1}^{M} \bar{P}_{\mathcal{N}_{i}} & 0 \\
-\sum_{i=1}^{M} \bar{P}_{\mathcal{N}_{i}}\left(A+B K_{t}\right) & -\sum_{i=1}^{M} \bar{P}_{\mathcal{N}_{i}} & 0 \\
0 & 0 & \sum_{i=1}^{M} \beta_{i}
\end{array}\right]-s_{0}\left[\begin{array}{ccc}
-\sum_{i=1}^{M} \bar{P}_{\mathcal{N}_{i}} & 0 & 0 \\
0 & 0 & 0 \\
0 & 0 & \sum_{i=1}^{M} \beta_{i}
\end{array}\right]} \\
& -\sum_{i=1}^{M} s_{i}\left[\begin{array}{ccc}
0 & 0 & 0 \\
0 & -\bar{I}_{i} & 0 \\
0 & 0 & v_{i}
\end{array}\right] \geq 0 \\
& {\left[\begin{array}{ccc}
-\left(A+B K_{t}\right)^{T} \bar{P}_{\mathcal{N}_{i}}\left(A+B K_{t}\right) & -\left(A+B K_{t}\right)^{T} \bar{P}_{\mathcal{N}_{i}} & 0 \\
-\bar{P}_{\mathcal{N}_{i}}\left(A+B K_{t}\right) & -\bar{P}_{\mathcal{N}_{i}} & 0 \\
0 & 0 & \beta_{i}
\end{array}\right]-s_{0}\left[\begin{array}{ccc}
-\bar{P}_{\mathcal{N}_{i}} & 0 & 0 \\
0 & 0 & 0 \\
0 & 0 & \beta_{i}
\end{array}\right]-s_{i}\left[\begin{array}{ccc}
0 & 0 & 0 \\
0 & -\bar{I}_{i} & 0 \\
0 & 0 & v_{i}
\end{array}\right] \geq \bar{S}_{i}, \forall i \in\{1, \ldots, M\}}
\end{aligned}
$$

set, i.e. $\overline{\mathcal{U}}_{k}=\left\{u \in \mathbb{R}^{p} \mid H u \leq \bar{h}_{k}\right\}$, can thus be obtained by modifying every single half space constraint as

$$
\begin{aligned}
& \bar{g}_{k, j}=g_{j}-\sigma_{\mathcal{W}}\left(G_{j}^{T}, k\right), \forall j \in\{1, \ldots, q\} \\
& \bar{h}_{k, l}=h_{l}-\sigma_{\mathcal{W}}\left(K_{t}^{T} H_{l}^{T}, k\right), \forall l \in\{1, \ldots, r\} .
\end{aligned}
$$

The evaluations of the $k$-step support function in the computation of the differences in (31) and (32) can be done by distributed optimization. This becomes apparent when considering the fact that the optimization problem (30) in the definition of the $k$-step support function has the same coupling structure as a regular distributed MPC problem.

\section{Robust MPC: Distributed Closed-Loop OPERATION}

In this section it is shown that closed-loop operation of both robust MPC controllers described in Section III can be performed by distributed optimization.

\section{A. Closed-loop Operation of Robust Distributed MPC based on [11]}

A characteristic of the robust MPC approach proposed in [11] is the fact that due to constraint (7b), the nominal initial state is not equal to the current state of the system. Constraint (7b) is structured and can be handled by distributed optimization as suggested in [18]. Following [18] might however result in prohibitively large local problems for large networks of subsystems. Therefore, in this section, a sufficient condition for constraint (7b) to hold is presented. This condition is based on local coupling only. In the following, $x(t)$ denotes the system state at time $t$ and $x_{r 1}^{*}(t+k \mid t)$ denotes the $k$ 'th element of the optimal state sequence to problem (7) at $t$.

Consider a structured RPI set $\mathcal{Z}=\{x \in$ $\left.\mathbb{R}^{m} \mid \sum_{i=1}^{M} x_{\mathcal{N}_{i}}^{T} P x_{\mathcal{N}_{i}} \leq 1\right\}$ and functions $\beta_{i}(\cdot): \mathbb{N} \rightarrow[0,1]$, which are used to define sets $\mathcal{Z}_{i}(t)=\left\{x_{\mathcal{N}_{i}} \in\right.$ $\left.\mathbb{R}^{m_{\mathcal{N}_{i}}} \mid \sum_{i=1}^{M} x_{\mathcal{N}_{i}}^{T} P_{\mathcal{N}_{i}} x_{\mathcal{N}_{i}} \leq \beta_{i}(t)\right\}$, where

$$
\forall t \in \mathbb{N}: \sum_{i=1}^{M} \beta_{i}(t) \leq 1 .
$$

Consequently, constraint (7b) can be enforced at time $t$ by $M$ local constraints of the form

$$
\forall i \in\{1, \ldots, M\}: x_{\mathcal{N}_{i}}(t \mid t) \in x_{\mathcal{N}_{i}}(t) \oplus \mathcal{Z}_{i}(t)
$$

These constraints couple neighboring subsystems locally, the global coupling is removed. A strategy on how to define functions $\beta_{i}(t)$ such that problem (7) remains recursively feasible is stated in the following theorem.

Theorem V.1 Given $\forall i \in\{1, \ldots, M\}$ functions $\beta_{i}(\cdot)$ as

$$
\begin{aligned}
\beta_{i}(t):=\left(x_{\mathcal{N}_{i}}(t)-\right. & \left.x_{\mathcal{N}_{i}, r 1}^{*}(t \mid t-1)\right)^{T} P_{\mathcal{N}_{i}} . \\
& \left(x_{\mathcal{N}_{i}}(t)-x_{\mathcal{N}_{i}, r 1}^{*}(t \mid t-1)\right)
\end{aligned}
$$

with $\beta_{i}(0)>0$ and $\sum_{i=1}^{M} \beta_{i}(0) \leq 1$, problem (7) remains feasible $\forall t \geq 1$ if (7b) is replaced by (34).

Proof: Theorem III.2 holds under recursive feasibility of the shifted nominal trajectory of problem (7). Here we show recursive feasibility under (34) instead of (7b). Given constraint (34) and $\sum_{i=1}^{M} \beta_{i}(t) \leq 1$ are satisfied at time $t$, we know that $\sum_{i=1}^{M}\left(x_{\mathcal{N}_{i}}(t)-x_{\mathcal{N}_{i}, r 1}^{*}(t \mid t)\right)^{T} P_{\mathcal{N}_{i}}\left(x_{\mathcal{N}_{i}}(t)-\right.$ $\left.x_{\mathcal{N}_{i}, r 1}^{*}(t \mid t)\right) \leq \sum_{i=1}^{M} \beta_{i}(t) \leq 1$ and thus $x(t) \in x_{r 1}^{*}(t \mid t) \oplus \mathcal{Z}$. Since $\mathcal{Z}$ is an RPI set, by Proposition 1 in [11] we know that $x(t+1) \in x_{r 1}^{*}(t+1 \mid t) \oplus \mathcal{Z}$. Thus, we obtain

$$
z^{T} P z=\sum_{i=1}^{M} z_{i}^{T} P_{\mathcal{N}_{i}} z_{i}=\sum_{i=1}^{M} \beta_{i}(t+1) \leq 1,
$$

where $z_{i}:=\left(x_{\mathcal{N}_{i}}(t+1)-x_{\mathcal{N}_{i}, r 1}^{*}(t+1 \mid t)\right)$ and $z:=(x(t+$ $\left.1)-x_{r 1}^{*}(t+1 \mid t)\right)$. Thus, the shifted trajectory is feasible for constraint (34).

\section{B. Closed-loop Operation of Robust Distributed MPC based on [15]}

During closed-loop operation, problem (12) has to be solved by distributed optimization in order to evaluate the control law $\kappa_{r 2}(x)$. This operation exhibits the same complexity as a nominal distributed MPC problem and can thus be solved by standard distributed optimization techniques.

\section{NUMERICAL EXAMPLE}

\section{A. Example Setup}

The system considered is a ring of 5 random second order systems which are coupled in a ring topology, hence $\mathcal{N}_{1}=$ $\{5,1,2\}, \mathcal{N}_{5}=\{4,5,1\}$ and $\mathcal{N}_{i}=\{i-1, i, i+1\}$ for $i \in\{2,3,4\}$. The dynamics of a subsystem $i$ is defined as

$$
x_{i}^{+}=A_{i i} x_{i}+\alpha \sum_{j \in \mathcal{N}_{i} \backslash i} A_{i j} x_{j}+B_{i} u_{i},
$$

where all entries in the matrices $A_{i i}, A_{i j}$ and $B_{i}$ are drawn from a uniform distribution within $[-0.5,0.5]$. The parameter $\alpha$ is a scalar real number by which the coupling strength in the system can be varied. The nominal state and input 
constraints are $\mathcal{X}=\left\{x \in \mathbb{R}^{10} \mid\|x\|_{\infty} \leq 2\right\}$ and $\mathcal{U}=\{u \in$ $\left.\mathbb{R}^{5} \mid\|u\|_{\infty} \leq 10\right\}$ and for each subsystem the local additive disturbance lies in the ball $\mathcal{W}=\left\{w \in \mathbb{R}^{2} \mid w^{T} w \leq 0.1\right\}$. The prediction horizon is $N=5$ and the quadratic stage cost penalizes the state twice as much as the input.

The following setups are compared: Distr. robust MPC based on [11] with global coupling constraint (Mayne1), distr. robust MPC based on [11] with the online heuristic discussed in Section V-A (Mayne2), distr. robust MPC based on [15] (Chisci) and the non-iterative MPC controller proposed in [6] (Farina), where the original formulation of [6] was modified to capture locally additive disturbance. Thus, the presented example provides both a comparison between various distributed robust MPC approaches and a comparison between robust iterative and non-iterative distributed MPC.

By choosing $\alpha=0.3$, a low coupling example is obtained. The closed-loop costs over 20 simulation steps of the different setups are illustrated in the box-plot in Fig. 1, where every box accumulates the costs corresponding to 10 different initial states, simulated for the same set of 3 different disturbance realizations. It is apparent, that all iterative distributed MPC approaches perform equally, while the non-iterative approach performs significantly worse.

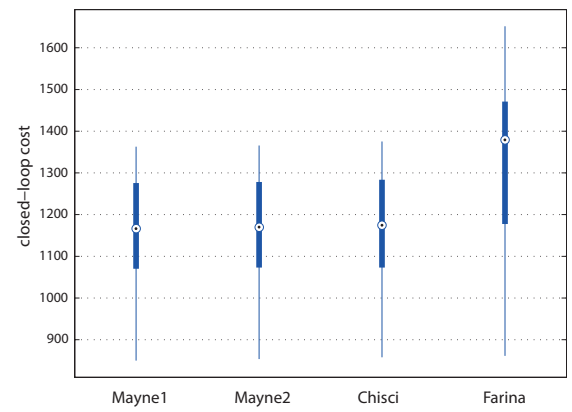

Fig. 1: Accumulated closed-loop costs, $\alpha=0.3$.

For stronger coupling, the synthesis procedure, especially for the non-iterative case, becomes more difficult. Tab. I denotes the largest values of $\alpha$, for which the synthesis problems for the different setups are feasible. It is apparent that the iterative approaches are viable for much larger coupling than the non-iterative ones. The closed-loop costs

\begin{tabular}{|c|c|c|c|c|}
\hline & Mayne1 & Mayne2 & Chisci & Farina \\
\hline$\alpha$ & 2.6 & 2.6 & 2.6 & 0.39 \\
\hline
\end{tabular}

TABLE I: Max. $\alpha$ for problems synthesis to be feasible.

of the iterative approaches under $\alpha=2.5$ are illustrated in the box-plot in Fig. 2. The controllers based on [11] have significantly lower closed-loop cost than that based on [15]. This is likely due to the fact that in [11] the nominal initial state can be chosen flexibly, which might allow for better adjustment to disturbances. Furthermore, the setup Mayne1 results in less outliers than the other setups.

\section{REFERENCES}

[1] A. N. Venkat, I. A. Hiskens, J. B. Rawlings, and S. J. Wright, "Distributed MPC strategies with application to power system au-

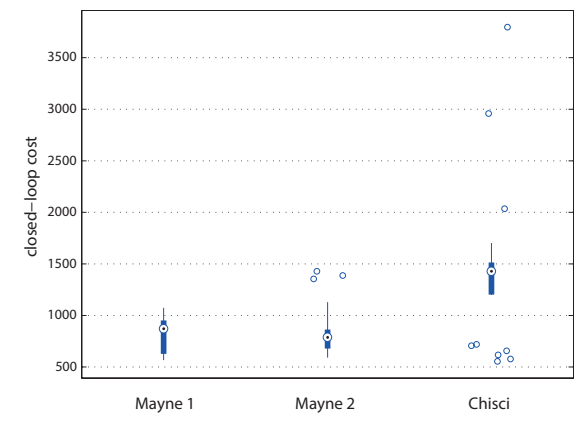

Fig. 2: Accumulated closed-loop costs, $\alpha=2.5$.

tomatic generation control," IEEE Transactions on Control Systems Technology, vol. 16, no. 6, pp. 1192-1206, 2008.

[2] R. R. Negenborn, P. J. van Overloop, T. Keviczky, and B. D. Schutter, "Distributed model predictive control of irrigation canals," Networks and Heterogeneous Media (NHM), vol. 4, no. 2, pp. 359-380, 2009.

[3] A. N. Venkat, J. B. Rawlings, and S. J. Wright, "Stability and optimality of distributed model predictive control," in 44th IEEE Conference on Decisions and Control, Seville, Spain, 2005, pp. 6680 $-6685$.

[4] Y. Wakasa, M. Arakawa, K. Tanaka, and T. Akashi, "Decentralized model predictive control via dual decomposition," in Proceedings of the 47th Conference on Decision and Control, Cancun, Mexico, 2008.

[5] D. Jia and B. H. Krogh, "Distributed model predictive control," in Proceedings of the American Control Conference, Arlington, 2001, pp. $2767-2772$.

[6] M. Farina and R. Scattolini, "Distributed predictive control: A noncooperative algrorithm with neighbor-to-neighbor communication for linear systems," Automatica, vol. 48, no. 6, pp. 1088-1096, June 2012.

[7] P. Giselsson and A. Rantzer, "Distributed model predictive control with suboptimality and stability guarantees," in 49th IEEE Conference on Decision and Control, Atlanta, GA, USA, 2010.

[8] M. D. Doan, T. Keviczky, and B. D. Schutter, "A distributed optimization-based approach for hierarchical mpc of large-scale systems with coupled dynamics and constraints," in 50th IEEE Conference on Decision and Control and European Control Conference (CDC-ECC), 2011, pp. 5236-5241.

[9] C. Conte, N. R. Voellmy, M. N. Zeilinger, M. Morari, and C. N. Jones, "Distributed synthesis and control of constrained linear systems," in Proceeding of the American Control Conference, Montreal, Canada, June 2012, pp. 6017-6022.

[10] A. Bemporad, F. Borrelli, and M. Morari, "Min-max control of constrained uncertain discrete-time linear systems," IEEE Transactions on Automatic Control, vol. 48, no. 9, pp. 1600-1606, September 2003.

[11] D. Q. Mayne, M. M. Seron, and S. V. Raković, "Robust model predictive control of constrained linear systems with bounded disturbances," Automatica, 2005.

[12] P. Trodden and A. Richards, "Distributed model predictive control of linear systems with persistent disturbances," International Journal of Control, vol. 83, no. 8, pp. 1653-1663, 2010.

[13] D. Q. Mayne, J. B. Rawlings, C. V. Rao, and P. O. M. Scokaert, "Constrained model predictive control: Stability and optimality," $\mathrm{Au}$ tomatica, vol. 36, pp. 789-814, 2000.

[14] D. P. Bertsekas and J. N. Tsitsiklis, Parallel and Distributed Computation. Old Tappan, NJ (USA): Prentice Hall Inc., 1989.

[15] L. Chisci, J. A. Rossiter, and G. Zappa, "Systems with persistent disturbances: Predictive control with restricted constraints," Automatica, vol. 37, pp. 1019-1028, 2001.

[16] S. V. Rakovic, E. C. Kerrigan, K. I. Kouramas, and D. Q. Mayne, "Invariant approximations of the minimal robust positively invariant set," IEEE Transactions on Automatic Control, vol. 50, no. 3, pp. 406410, 2005.

[17] S. Boyd, L. E. Ghaoui, E. Feron, and V. Balakrishnan, Linear Matrix Inequalities in System and Control Theory. Society for Industrial and Applied Mathematics (SIAM), 1994, vol. 15.

[18] I. D. Schizas, A. Ribeiro, and G. B. Giannakis, "Consensus in ad hoc wsns with noisy links - part i: Distributed estimation of deterministic signals," IEEE Transactions on Signal Processing, vol. 56, no. 1, pp. 350-364, January 2008. 